\title{
A METHOD FOR COMPUTING TOTAL DOWNTIME DISTRIBUTIONS IN REPAIRABLE SYSTEMS
}

\author{
SUYONO, ${ }^{*}$ Universitas Negeri Jakarta \\ J. A. M. VAN DER WEIDE, ${ }^{* *}$ Delft University of Technology
}

\begin{abstract}
In this paper we derive the distribution of the total downtime of a repairable system during a given time interval. We allow dependence of the failure time and the repair time. The results are presented in the form of Laplace transforms which can be inverted numerically. We also discuss asymptotic properties of the total downtime.
\end{abstract}

Keywords: Downtime; Laplace transform; Laplace-Stieltjes transform; point process

AMS 2000 Subject Classification: Primary 60E10

Secondary 60G55; 60F05; 60K05

\section{Introduction}

Consider a repairable system which is at any time either in operation (up) or under repair (down) after failure. The effectiveness of the system can be measured by the total uptime (or downtime), i.e. the total amount of time the system is up (or down) during a given time interval. An expression for the cumulative distribution function (CDF) of the total downtime up to a given time $t$ has been derived by several authors using different methods. Takács [9] used a set theoretic method. The derivation of Muth [5] is based on consideration of the excess time. Finally, Funaki and Yoshimoto [3] derived the CDF of the total downtime by a conditioning technique. Srinivasan et al. [8] derived an expression for the probability density function (PDF) of the total uptime of the system up to time $t$. They also discussed its covariance structure. For a longer time interval, Takács [9] and Rényi [6] proved that the distribution of the total downtime approaches a normal distribution. Takács [9] also discussed asymptotic mean and variance of the total downtime. In all these papers it is assumed that the failure time and the repair time are independent.

In this paper we use a different method for computation of the distribution of the total downtime. We also consider a more general situation where we allow dependence of the failure time and the repair time. Our derivation is based on a representation of the total downtime as a functional of a Poisson point process.

This paper is organized as follows. In Section 2 we define the total downtime and derive its distribution in a fixed time interval. In Section 3 we study the covariance structure and asymptotic properties of the total downtime and in Section 4 we give an example.

Received 13 September 2002; revision received 3 February 2003.

* Postal address: Jurusan Matematika, Universitas Negeri Jakarta, Jl. Pemuda 10, Rawamangun Jakarta Timur 13200, Indonesia.

** Postal address: Delft University of Technology, HB 06.130, Mekelweg 4, 2628 CD Delft, The Netherlands.

Email address: j.a.m.vanderweide@its.tudelft.nl 


\section{Distribution of total downtime}

We consider a repairable system which is at any time either in operation (up) or under repair (down) after failure, denoted as 1 and 0 respectively. Suppose that the system starts to operate at time $t=0$. Let $\left(X_{i}\right)$ and $\left(Y_{i}\right), i \geq 1$, denote the time spent in the states 1 and 0 respectively during the $i$ th visit to that state. The random variables $X_{i}$ and $Y_{i}$ are known as the failure time and the repair time respectively. We assume that the sequence $\left(X_{i}, Y_{i}\right)$ of random vectors is independent and identically distributed (i.i.d.) with strictly positive components. However, our set-up is more general than that in [3], [5], [6], [8], [9], as we allow $X_{i}$ and $Y_{i}$ to be dependent.

Let $S_{n}=\sum_{i=1}^{n}\left(X_{i}+Y_{i}\right)$ for $n \geq 1$ and $S_{0} \equiv 0$, and let $N(t)=\sup \left\{n \geq 0: S_{n} \leq t\right\}$. Then the total downtime $D(t)$ can be expressed (with the usual convention that the empty sum equals 0 ) as

$$
D(t)= \begin{cases}\sum_{i=1}^{N(t)} Y_{i} & \text { if } S_{N(t)} \leq t<S_{N(t)}+X_{N(t)+1}, \\ t-\sum_{i=1}^{N(t)+1} X_{i} & \text { if } S_{N(t)}+X_{N(t)+1} \leq t<S_{N(t)+1} .\end{cases}
$$

Denote the state of the system at time $t$ by $Z(t)$. We assume that $Z(t)$ is right continuous. Then the total downtime $D(t)$ can also be expressed as

$$
D(t)=\int_{0}^{t} \mathbf{1}_{\{0\}}(Z(s)) \mathrm{d} s .
$$

Throughout this paper we will use the following notation for CDFs:

$$
\begin{aligned}
F(x) & =\mathrm{P}\left(X_{1} \leq x\right), \\
G(y) & =\mathrm{P}\left(Y_{1} \leq y\right), \\
H(x, y) & =\mathrm{P}\left(X_{1} \leq x, Y_{1} \leq y\right), \\
K(w) & =\mathrm{P}\left(X_{1}+Y_{1} \leq w\right) .
\end{aligned}
$$

We write $F_{n}$ and $G_{n}$ for the CDFs of $\sum_{i=1}^{n} X_{i}$ and $\sum_{i=1}^{n} Y_{i}$ respectively. The Laplace-Stieltjes transforms of a CDF $F$ and a joint CDF $H$ will be denoted by $F^{*}$ and $H^{*}$ respectively, i.e. for $\alpha, \beta>0$,

and

$$
F^{*}(\alpha)=\int_{0}^{\infty} \mathrm{e}^{-\alpha x} \mathrm{~d} F(x)
$$

$$
H^{*}(\alpha, \beta)=\int_{0}^{\infty} \int_{0}^{\infty} \mathrm{e}^{-(\alpha x+\beta y)} \mathrm{d} H(x, y) .
$$

We will use point processes for the derivation of the distribution of the total downtime $D(t)$. Let $(\Omega, \mathcal{F}, \mathrm{P})$ be the probability space on which the i.i.d. sequence $\left(X_{i}, Y_{i}\right)$ is defined and also an i.i.d. sequence $\left(U_{i}, i \geq 1\right)$ of exponentially distributed random variables with parameter 1 such that the sequences $\left(U_{i}\right)$ and $\left(X_{i}, Y_{i}\right)$ are independent. Let $\left(T_{n}, n \geq 1\right)$ be the sequence of partial sums of the variables $U_{i}$. Then the map

$$
\Phi: \omega \mapsto \sum_{n=1}^{\infty} \delta_{\left(T_{n}(\omega), X_{n}(\omega), Y_{n}(\omega)\right)},
$$


where $\delta_{(x, y, z)}$ is the Dirac measure in $(x, y, z)$, defines a Poisson point process on $E=[0, \infty) \times$ $[0, \infty) \times[0, \infty)$ with intensity measure $v(\mathrm{~d} t \mathrm{~d} x \mathrm{~d} y)=\mathrm{d} t \mathrm{~d} H(x, y)$; see e.g. [7, p. 135]. Note that, for almost all $\omega \in \Omega, \Phi(\omega)$ is a simple point measure on $E$ such that there is at most one point from the support of $\Phi(\omega)$ on each set $\{t\} \times[0, \infty) \times[0, \infty)$. Let $M_{p}(E)$ be the set of all point measures on $E$. We will denote by $\mathrm{P}_{v}$ the distribution of $\Phi$ over $M_{p}(E)$.

For $t \in[0, \infty)$, define on $M_{p}(E)$ the functionals

$$
\begin{aligned}
& A_{X}(t)(\mu):=\int_{E} x \mathbf{1}_{[0, t)}(s) \mu(\mathrm{d} s \mathrm{~d} x \mathrm{~d} y), \\
& A_{Y}(t)(\mu):=\int_{E} y \mathbf{1}_{[0, t)}(s) \mu(\mathrm{d} s \mathrm{~d} x \mathrm{~d} y),
\end{aligned}
$$

and

$$
A(t)(\mu):=A_{X}(t)(\mu)+A_{Y}(t)(\mu) .
$$

So, for example, $A(t)(\mu)$ is the sum of the $x$ - and the $y$-coordinates of the points in the set of $\operatorname{supp} \mu \cap[0, t] \times[0, \infty) \times[0, \infty)$, where supp $\mu=\{(s, x, y): \mu\{(s, x, y)\}>0\}$. In the sequel we will write $A_{X}(t, \mu)$ for $A_{X}(t)(\mu)$ and similarly for $A_{Y}(t)(\mu)$ and $A(t)(\mu)$. Define also for $t \geq 0$

$$
\begin{aligned}
B(t)(\mu):=\int_{E}\left\{\mathbf{1}_{[0, x)}(t-A(s, \mu)) A_{Y}(s, \mu)\right. \\
\\
\left.+\mathbf{1}_{[x, x+y)}(t-A(s, \mu))\left[t-A_{X}(s+, \mu)\right]\right\} \mu(\mathrm{d} s \mathrm{~d} x \mathrm{~d} y),
\end{aligned}
$$

where $A_{X}(s+, \mu)=\int_{E} x \mathbf{1}_{[0, s]}(r) \mu(\mathrm{d} r \mathrm{~d} x \mathrm{~d} y)$. In the next lemma we explain the meaning of $B(t)$.

Lemma 1. With probability 1,

$$
D(t)=B(t)(\Phi)
$$

Proof. Let $\omega \in \Omega$ such that $\Phi(\omega)$ is a simple point measure on $E$ with at most one point of $\operatorname{supp} \mu$ on each set $\{t\} \times[0, \infty) \times[0, \infty)$. As noted before, the set of these points has probability 1 . Then

$$
\begin{aligned}
B(t)(\Phi(\omega))=\sum_{i=1}^{\infty} & \left\{\mathbf{1}_{\left[0, X_{i}(\omega)\right)}\left(t-A\left(T_{i}(\omega), \Phi(\omega)\right)\right) A_{Y}\left(T_{i}(\omega), \Phi(\omega)\right)\right. \\
& \left.+\mathbf{1}_{\left[X_{i}(\omega), X_{i}(\omega)+Y_{i}(\omega)\right)}\left(t-A\left(T_{i}(\omega), \Phi(\omega)\right)\right)\left[t-A_{X}\left(T_{i}(\omega)+, \Phi(\omega)\right)\right]\right\} .
\end{aligned}
$$

Note that

$$
\mathbf{1}_{\left[0, X_{i}(\omega)\right)}\left(t-A\left(T_{i}(\omega), \Phi(\omega)\right)\right)=1
$$

implies that $i=N(t, \omega)+1$. Similarly, if

$$
\mathbf{1}_{\left[X_{i}(\omega), X_{i}(\omega)+Y_{i}(\omega)\right)}\left(t-A\left(T_{i}(\omega), \Phi(\omega)\right)\right)=1,
$$

then $i=N(t, \omega)+1$. Since the intervals

$$
\left\{\left[S_{i-1}, S_{i-1}+X_{i}\right),\left[S_{i-1}+X_{i}, S_{i-1}+X_{i}+Y_{i}\right): i \geq 1\right\}
$$

partition $[0, \infty)$, for any $t>0$ one and only one of the indicators in the sum will be nonzero. So, if

$$
\mathbf{1}_{\left[0, X_{i}(\omega)\right)}\left(t-A\left(T_{i}(\omega), \Phi(\omega)\right)\right)=1,
$$


then $i=N(t, \omega)+1$ and

$$
\begin{aligned}
B(t)(\Phi(\omega)) & =A_{Y}\left(T_{N(t, \omega)+1}(\omega), \Phi(\omega)\right) \\
& = \begin{cases}0 & \text { if } N(t, \omega)=0, \\
\sum_{j=1}^{N(t, \omega)} Y_{j}(\omega) & \text { if } N(t, \omega) \geq 1,\end{cases}
\end{aligned}
$$

and, if

$$
\mathbf{1}_{\left[X_{i}(\omega), X_{i}(\omega)+Y_{i}(\omega)\right)}\left(t-A\left(T_{i}(\omega), \Phi(\omega)\right)\right)=1,
$$

then

$$
\begin{aligned}
B(t)(\Phi(\omega)) & =t-A_{X}\left(T_{N(t, \omega)+1}(\omega)+, \Phi(\omega)\right) \\
& =t-\sum_{j=1}^{N(t, \omega)+1} X_{j}(\omega) .
\end{aligned}
$$

The following theorem gives the distribution of the total downtime $D(t)$ in the form of a double Laplace transform.

Theorem 1. For $\alpha, \beta>0$,

$$
\int_{0}^{\infty} \mathrm{E}\left[\mathrm{e}^{-\alpha D(t)}\right] \mathrm{e}^{-\beta t} \mathrm{~d} t=\frac{\alpha\left[1-F^{*}(\beta)\right]+\beta\left[1-H^{*}(\beta, \alpha+\beta)\right]}{\beta(\alpha+\beta)\left[1-H^{*}(\beta, \alpha+\beta)\right]} .
$$

Proof. By Lemma 1 and using Fubini's theorem, we obtain that

$$
\begin{aligned}
& \int_{0}^{\infty} \mathrm{E}\left(\mathrm{e}^{-\alpha D(t)}\right) \mathrm{e}^{-\beta t} \mathrm{~d} t \\
&=\int_{0}^{\infty} \mathrm{d} t \int_{M_{p}(E)} \mathrm{P}_{\nu}(\mathrm{d} \mu) \exp \left\{-\alpha \int_{E} \mu(\mathrm{d} s \mathrm{~d} x \mathrm{~d} y)\left[\mathbf{1}_{[0, x)}(t-A(s, \mu)) A_{Y}(s, \mu)\right.\right. \\
&\left.\left.\quad+\mathbf{1}_{[x, x+y)}(t-A(s, \mu))\left(t-A_{X}(s+, \mu)\right)\right]\right\} \mathrm{e}^{-\beta t} \\
&=\int_{M_{p}(E)} \mathrm{P}_{\nu}(\mathrm{d} \mu) \int_{E} \mu(\mathrm{d} s \mathrm{~d} x \mathrm{~d} y) \int_{0}^{\infty} \mathrm{d} t\left[\mathbf{1}_{[0, x)}(t-A(s, \mu)) \mathrm{e}^{-\alpha A_{Y}(s, \mu)}\right. \\
&\left.\quad+\mathbf{1}_{[x, x+y)}(t-A(s, \mu)) \mathrm{e}^{-\alpha\left(t-A_{X}(s+, \mu)\right)}\right] \mathrm{e}^{-\beta t} \\
&=I_{1}+I_{2},
\end{aligned}
$$

where

$$
\begin{aligned}
& I_{1}=\int_{M_{p}(E)} \mathrm{P}_{v}(\mathrm{~d} \mu) \int_{E} \mu(\mathrm{d} s \mathrm{~d} x \mathrm{~d} y) \int_{0}^{\infty} \mathrm{d} t \mathbf{1}_{[0, x)}(t-A(s, \mu)) \mathrm{e}^{-\alpha A_{Y}(s, \mu)} \mathrm{e}^{-\beta t}, \\
& I_{2}=\int_{M_{p}(E)} \mathrm{P}_{\nu}(\mathrm{d} \mu) \int_{E} \mu(\mathrm{d} s \mathrm{~d} x \mathrm{~d} y) \int_{0}^{\infty} \mathrm{d} t \mathbf{1}_{[x, x+y)}(t-A(s, \mu)) \mathrm{e}^{-\alpha\left(t-A_{X}(s+, \mu)\right)} \mathrm{e}^{-\beta t} .
\end{aligned}
$$


Using the Palm formula and Laplace functional of Poisson point processes (see [4, p. 56] and [7, p. 130] respectively) we obtain that

$$
\begin{aligned}
I_{1} & =\frac{1}{\beta} \int_{M_{p}(E)} \mathrm{P}_{\nu}(\mathrm{d} \mu) \int_{E} \mu(\mathrm{d} s \mathrm{~d} x \mathrm{~d} y)\left[1-\mathrm{e}^{-\beta x}\right] \mathrm{e}^{-\left(\alpha A_{Y}(s, \mu)+\beta A(s, \mu)\right)} \\
& =\frac{1}{\beta} \int_{0}^{\infty} \mathrm{d} s \int_{0}^{\infty} \int_{0}^{\infty} \mathrm{d} H(x, y) \int_{M_{p}(E)} \mathrm{P}_{v}(\mathrm{~d} \mu)\left[1-\mathrm{e}^{-\beta x}\right] \mathrm{e}^{-\left(\alpha A_{Y}(s, \mu)+\beta A(s, \mu)\right)} \\
& =\frac{1}{\beta} \int_{0}^{\infty} \int_{0}^{\infty} \mathrm{d} H(x, y)\left[1-\mathrm{e}^{-\beta x}\right] \int_{0}^{\infty} \mathrm{d} s \int_{M_{p}(E)} \mathrm{P}_{\nu}(\mathrm{d} \mu) \\
& =\frac{1}{\beta}\left[1-F^{*}(\beta)\right] \int_{0}^{\infty} \mathrm{d} s \exp \left(-\int_{0}^{\infty} \mathrm{d} \tilde{s} \int_{0}^{\infty} \int_{0}^{\infty} \mathrm{d} H(\tilde{x}, \tilde{y})\left[1-\mathrm{e}^{-\mathbf{1}_{[0, s)}(\tilde{s})(\beta \tilde{x}+(\alpha+\beta) \tilde{y})}\right]\right) \\
& =\frac{1}{\beta}\left[1-F^{*}(\beta)\right] \int_{0}^{\infty} \exp \left(-s \int_{0}^{\infty} \int_{0}^{\infty} \mathrm{d} H(\tilde{x}, \tilde{y})\left[1-\mathrm{e}^{-(\beta \tilde{x}+(\alpha+\beta) \tilde{y})}\right]\right) \\
& =\frac{1-F^{*}(\beta)}{\beta\left[1-H^{*}(\beta, \alpha+\beta)\right]}
\end{aligned}
$$

and

$$
\begin{aligned}
& I_{2}=\int_{M_{p}(E)} \mathrm{P}_{v}(\mathrm{~d} \mu) \int_{E} \mu(\mathrm{d} s \mathrm{~d} x \mathrm{~d} y) \int_{x}^{x+y} \mathrm{~d} t \mathrm{e}^{-(\alpha+\beta) A(s, \mu)+\alpha A_{X}(s+, \mu)} \mathrm{e}^{-(\alpha+\beta) t} \\
& =\frac{1}{\alpha+\beta} \int_{0}^{\infty} \mathrm{d} s \int_{0}^{\infty} \int_{0}^{\infty} \mathrm{d} H(x, y) \int_{M_{p}(E)} \mathrm{P}_{\nu}(\mathrm{d} \mu)\left[\mathrm{e}^{-(\alpha+\beta) x}-\mathrm{e}^{-(\alpha+\beta)(x+y)}\right] \\
& \times \exp \left\{-(\alpha+\beta) A(s-, \mu)+\alpha\left[A_{X}(s+, \mu)+x\right]\right\} \\
& =\frac{1}{\alpha+\beta} \int_{0}^{\infty} \int_{0}^{\infty} \mathrm{d} H(x, y) \mathrm{e}^{-\beta x}\left[1-\mathrm{e}^{-(\alpha+\beta) y}\right] \int_{0}^{\infty} \mathrm{d} s \int_{M_{p}(E)} \mathrm{P}_{v}(\mathrm{~d} \mu) \\
& \times \exp \left\{-\int_{E} \mu(\mathrm{d} \tilde{s} \mathrm{~d} \tilde{x} \mathrm{~d} \tilde{y})\left[\mathbf{1}_{[0, s)}(\tilde{s})(\alpha+\beta)(\tilde{x}+\tilde{y})-\mathbf{1}_{[0, s]}(\tilde{s}) \alpha \tilde{x}\right]\right\} \\
& =\frac{1}{\alpha+\beta}\left[F^{*}(\beta)-H^{*}(\beta, \alpha+\beta)\right] \int_{0}^{\infty} \mathrm{d} s \\
& \times \exp \left\{-\int_{0}^{\infty} \mathrm{d} \tilde{s} \int_{0}^{\infty} \int_{0}^{\infty} \mathrm{d} H(\tilde{x}, \tilde{y})\right. \\
& \left.\times\left[1-\exp \left(-\left[\mathbf{1}_{[0, s)}(\tilde{s})(\alpha+\beta)(\tilde{x}+\tilde{y})-\mathbf{1}_{[0, s]}(\tilde{s}) \alpha \tilde{x}\right]\right)\right]\right\} \\
& =\frac{1}{\alpha+\beta}\left[F^{*}(\beta)-H^{*}(\beta, \alpha+\beta)\right] \int_{0}^{\infty} \mathrm{d} s \\
& \times \exp \left\{-s \int_{0}^{\infty} \int_{0}^{\infty}\left[1-\mathrm{e}^{-[\beta \tilde{x}+(\alpha+\beta) \tilde{y}]}\right] \mathrm{d} H(\tilde{x}, \tilde{y})\right\} \\
& =\frac{F^{*}(\beta)-H^{*}(\beta, \alpha+\beta)}{(\alpha+\beta)\left[1-H^{*}(\beta, \alpha+\beta)\right]} .
\end{aligned}
$$


Corollary 1. Taking derivatives with respect to $\alpha$ in (2) and setting $\alpha=0$, we get

$$
\int_{0}^{\infty} \mathrm{E}[D(t)] \mathrm{e}^{-\beta t} \mathrm{~d} t=\frac{F^{*}(\beta)-H^{*}(\beta, \beta)}{\beta^{2}\left[1-H^{*}(\beta, \beta)\right]}
$$

and

$$
\int_{0}^{\infty} \mathrm{E}\left[D(t)^{2}\right] \mathrm{e}^{-\beta t} \mathrm{~d} t=\frac{2}{\beta^{3}}\left[\frac{F^{*}(\beta)-H^{*}(\beta, \beta)}{1-H^{*}(\beta, \beta)}-\frac{\beta\left[1-F^{*}(\beta)\right] \mathrm{E}\left(Y_{1} \mathrm{e}^{-\beta\left(X_{1}+Y_{1}\right)}\right)}{\left[1-H^{*}(\beta, \beta)\right]^{2}}\right] .
$$

Remark 1. When $\left(X_{i}\right)$ and $\left(Y_{j}\right)$ are independent, (2) simplifies to

$$
\int_{0}^{\infty} \mathrm{E}\left(\mathrm{e}^{-\alpha D(t)}\right) \mathrm{e}^{-\beta t} \mathrm{~d} t=\frac{\alpha\left[1-F^{*}(\beta)\right]+\beta\left[1-F^{*}(\beta) G^{*}(\alpha+\beta)\right]}{\beta(\alpha+\beta)\left[1-F^{*}(\beta) G^{*}(\alpha+\beta)\right]} .
$$

For the independent case, Takács [9], Muth [5], Funaki and Yoshimoto [3] derived the following formula for the distribution function of the total downtime:

$$
\mathrm{P}(D(t) \leq x)= \begin{cases}\sum_{n=0}^{\infty} G_{n}(x)\left[F_{n}(t-x)-F_{n+1}(t-x)\right] & \text { for } t>x, \\ 1, & \text { for } t \leq x,\end{cases}
$$

where $F_{n}$ and $G_{n}$ are the CDFs of $\sum_{i=1}^{n} X_{i}$ and $\sum_{i=1}^{n} Y_{i}$ respectively. Taking double Laplace transforms on both sides of (6) we obtain (5).

Remark 2. We can also derive the Laplace transform of $\mathrm{E}[D(t)]$ without using point processes. From the definition of $D(t)$ (see (1)), it follows that

$$
\mathrm{E}[D(t)]=t-\int_{0}^{t} A_{11}(s) \mathrm{d} s
$$

where $A_{11}(t)=\mathrm{P}(Z(t)=1)$ is the availability of the system. Taking Laplace transforms on both sides of (7) gives

$$
\int_{0}^{\infty} \mathrm{E}[D(t)] \mathrm{e}^{-\beta t} \mathrm{~d} t=\frac{1}{\beta^{2}}-\frac{1}{\beta} \hat{A}_{11}(\beta)
$$

where $\hat{A}_{11}(\beta)=\int_{0}^{\infty} A_{11}(t) \mathrm{e}^{-\beta t} \mathrm{~d} t$. But $A_{11}(t)$ satisfies the following integral equation (see [1]):

$$
A_{11}(t)=\bar{F}(t)+\int_{0}^{t} \bar{F}(t-u) \mathrm{d} m(u)
$$

where $\bar{F}(t)=1-F(t)$ and $m(t)=\mathrm{E}[N(t)]$. Taking Laplace transforms on both sides of (8) we obtain that

$$
\hat{A}_{11}(\beta)=\frac{1}{\beta}\left[1-F^{*}(\beta)\right]\left[1+m^{*}(\beta)\right],
$$

where $m^{*}$ is the Laplace-Stieltjes transform of $m(t)$. Moreover, it is well known that

$$
m^{*}(\beta)=\frac{K^{*}(\beta)}{1-K^{*}(\beta)} .
$$


Combining (9) and (10) we obtain that

$$
\hat{A}_{11}(\beta)=\frac{1-F^{*}(\beta)}{\beta\left[1-K^{*}(\beta)\right]},
$$

and so

$$
\int_{0}^{\infty} \mathrm{E}[D(t)] \mathrm{e}^{-\beta t} \mathrm{~d} t=\frac{F^{*}(\beta)-K^{*}(\beta)}{\beta^{2}\left[1-K^{*}(\beta)\right]},
$$

which is in agreement with (3) since $K^{*}(\beta)=H^{*}(\beta, \beta)$.

\section{Covariance and asymptotic properties of $D(t)$}

We start by considering the covariance structure of $D(t)$. Let $U(t)=t-D(t)$ be the total uptime of the system. Obviously, $\operatorname{cov}\left(D\left(t_{1}\right), D\left(t_{2}\right)\right)=\operatorname{cov}\left(U\left(t_{1}\right), U\left(t_{2}\right)\right)$. So we might as well study $\operatorname{cov}\left(U\left(t_{1}\right), U\left(t_{2}\right)\right)$.

The double Laplace transform of $\mathrm{E}\left[U\left(t_{1}\right) U\left(t_{2}\right)\right]$ is given in the following proposition, which is a generalization of a result in [8].

Proposition 1. For $\alpha, \beta>0$,

$$
\int_{0}^{\infty} \int_{0}^{\infty} \mathrm{E}\left[U\left(t_{1}\right) U\left(t_{2}\right)\right] \mathrm{e}^{-\alpha t_{1}-\beta t_{2}} \mathrm{~d} t_{1} \mathrm{~d} t_{2}=\frac{1}{\alpha \beta}[\hat{\varphi}(\alpha, \beta)+\hat{\varphi}(\beta, \alpha)]
$$

where

$$
\begin{aligned}
\hat{\varphi}(\alpha, \beta)= & \frac{\alpha\left[1-F^{*}(\beta)\right]-\beta\left[F^{*}(\beta)-F^{*}(\alpha+\beta)\right]}{\alpha \beta(\alpha+\beta)\left[1-K^{*}(\alpha+\beta)\right]} \\
& +\frac{\left[1-F^{*}(\beta)\right]\left[H^{*}(\beta, \beta)-H^{*}(\alpha+\beta, \beta)\right]}{\alpha \beta\left[1-H^{*}(\beta)\right]\left[1-K^{*}(\alpha+\beta)\right]} .
\end{aligned}
$$

Proof. First, it can easily be verified that, for $0 \leq t_{1} \leq t_{2}<\infty$,

$$
\mathrm{E}\left[U\left(t_{1}\right) U\left(t_{2}\right)\right]=2 \int_{x=0}^{t_{1}} \int_{y=x}^{t_{1}} \varphi(x, y) \mathrm{d} y \mathrm{~d} x+\int_{x=0}^{t_{1}} \int_{y=t_{1}}^{t_{2}} \varphi(x, y) \mathrm{d} y \mathrm{~d} x,
$$

where $\varphi(x, y)=\mathrm{P}(Z(x)=1, Z(y)=1)$. From this equation we obtain that

$$
\int_{t_{1}=0}^{\infty} \int_{t_{2}=t_{1}}^{\infty} \mathrm{E}\left[U\left(t_{1}\right) U\left(t_{2}\right)\right] \mathrm{e}^{-\alpha t_{1}-\beta t_{2}} \mathrm{~d} t_{2} \mathrm{~d} t_{1}=\frac{\hat{\varphi}(\alpha, \beta)}{\alpha \beta}+\frac{[\alpha-\beta] \hat{\varphi}(0, \alpha+\beta)}{\alpha \beta(\alpha+\beta)},
$$

where $\hat{\varphi}(\alpha, \beta)=\int_{0}^{\infty} \int_{0}^{\infty} \varphi(x, y) \mathrm{e}^{-\alpha x-\beta y} \mathrm{~d} x \mathrm{~d} y$.

Now we want to prove that $\hat{\varphi}(\alpha, \beta)$ satisfies (12). For $0 \leq x \leq y<\infty$,

$$
\begin{aligned}
\varphi(x, y)= & \mathrm{P}\left(Z(x)=1, Z(y)=1, y<X_{1}\right) \\
& +\mathrm{P}\left(Z(x)=1, Z(y)=1, x<X_{1}<y\right) \\
& +\mathrm{P}\left(Z(x)=1, Z(y)=1, X_{1}<x\right) .
\end{aligned}
$$

Obviously, $\mathrm{P}\left(Z(x)=1, Z(y)=1, y<X_{1}\right)=1-F(y)$. For the second term, note that the event

$$
\left\{Z(x)=1, Z(y)=1, x<X_{1}<y\right\}
$$


is equivalent to the event

$$
\left\{x<X_{1} \text { and, for some } n \geq 1, S_{n}<y<S_{n}+X_{n+1}\right\},
$$

where $S_{n}=\sum_{i=1}^{n}\left(X_{i}+Y_{i}\right)$. Let $R_{n}=\sum_{i=2}^{n}\left(X_{i}+Y_{i}\right)$ for $n \geq 2$. Then $\left(X_{1}, Y_{1}\right), R_{n}$ and $X_{n+1}$ are independent. Using this fact we can prove that

$$
\mathrm{P}\left(Z(x)=1, Z(y)=1, x<X_{1}<y\right)=\int_{x_{1} \in(x, y]} \int_{w \in\left[x_{1}, y\right]} A_{11}(y-w) \mathrm{d} H\left(x_{1}, w-x_{1}\right),
$$

where $A_{11}(t)$ denotes the availability of the system at time $t$ starting in state 1 at time 0 . Finally, the last term in (14) can be obtained by conditioning on $X_{1}+Y_{1}$ which gives

$$
\mathrm{P}\left(Z(x)=1, Z(y)=1, X_{1} \leq x\right)=\int_{0}^{x} \varphi(x-w, y-w) \mathrm{d} K(w) .
$$

Taking the double Laplace transform on both sides of (14) and using (11), (12) follows.

Finally, for $0 \leq t_{2} \leq t_{1}$, we simply interchange $\alpha$ and $\beta$ in (13) so that the proposition follows.

Now we want to address asymptotic properties of the total downtime $D(t)$. To this end, we use a method of Takács [9] which is based on a comparison with the asymptotic properties of a delayed renewal process related to the process that we are studying. We will use the following notation:

$$
\begin{gathered}
\mu_{X}=\mathrm{E}\left(X_{1}\right), \quad \mu_{Y}=\mathrm{E}\left(Y_{1}\right), \\
\sigma_{X}^{2}=\operatorname{var}\left(X_{1}\right), \quad \sigma_{Y}^{2}=\operatorname{var}\left(Y_{1}\right), \quad \sigma_{X Y}=\operatorname{cov}\left(X_{1}, Y_{1}\right) .
\end{gathered}
$$

Theorem 2. If $\mu_{X}+\mu_{Y}<\infty$, then

$$
\lim _{t \rightarrow \infty} \frac{\mathrm{E}[D(t)]}{t}=\frac{\mu_{Y}}{\mu_{X}+\mu_{Y}} .
$$

If $\sigma_{X}^{2}$ and $\sigma_{Y}^{2}$ are finite and $X_{1}+Y_{1}$ is a nonlattice random variable, then

$$
\lim _{t \rightarrow \infty}\left(\mathrm{E}[D(t)]-\frac{\mu_{Y} t}{\mu_{X}+\mu_{Y}}\right)=\frac{\mu_{Y} \sigma_{X}^{2}-\mu_{X} \sigma_{Y}^{2}-2 \mu_{X} \sigma_{X Y}}{2\left(\mu_{X}+\mu_{Y}\right)^{2}}-\frac{\mu_{X} \mu_{Y}}{2\left(\mu_{X}+\mu_{Y}\right)}
$$

and

$$
\lim _{t \rightarrow \infty} \frac{\operatorname{var}[D(t)]}{t}=\frac{\mu_{X}^{2} \sigma_{Y}^{2}+\mu_{Y}^{2} \sigma_{X}^{2}-2 \mu_{X} \mu_{Y} \sigma_{X Y}}{\left(\mu_{X}+\mu_{Y}\right)^{3}} .
$$

Proof. Let $\tilde{N}(t)$ be the delayed renewal process determined by the random variables $\left(V_{n}\right)$, $n=0,1,2, \ldots$, where $V_{0}$ has the distribution $\mathrm{P}\left(V_{0} \leq x\right)=\left(1 / \mu_{X}\right) \int_{0}^{x}[1-F(y)] \mathrm{d} y$ for $x \geq 0$ and $\mathrm{P}\left(V_{0} \leq x\right)=0$ otherwise, and

$$
V_{n}=X_{n}+Y_{n}, \quad n=1,2,3 \ldots
$$

Then using (3) and Laplace-Stieltjes-transform arguments, we can prove that

$$
\mathrm{E}[D(t)]+\mu_{X} \mathrm{E}[\tilde{N}(t)]=t .
$$

Since $\lim _{t \rightarrow \infty} \mathrm{E}[\tilde{N}(t)] / t=1 /\left(\mu_{X}+\mu_{Y}\right)$, the first part of the theorem follows. 
To prove (16), from (18) we obtain that

$$
\lim _{t \rightarrow \infty}\left(\mathrm{E}[D(t)]-\frac{\mu_{Y} t}{\mu_{X}+\mu_{Y}}\right)=-\mu_{X} \lim _{t \rightarrow \infty}\left(\mathrm{E}[\tilde{N}(t)]-\frac{t}{\mu_{X}+\mu_{Y}}\right) .
$$

Now, if $X_{1}+Y_{1}$ is a nonlattice random variable, then using formula (33) of [9] and by noting that $\mathrm{E}\left(V_{0}\right)=\left(\sigma_{X}^{2}+\mu_{X}^{2}\right) / 2 \mu_{X},(16)$ follows.

To prove asymptotic variance of $D(t)$, we construct another delayed renewal process as follows. Let $\breve{N}(t)$ be the delayed renewal process determined by the random variables $\left(V_{n}\right)$, $n=0,1,2, \ldots$, where $V_{0}$ has Laplace transform

$$
\mathrm{E}\left(\mathrm{e}^{-\beta V_{0}}\right)=\frac{\left[1-F^{*}(\beta)\right] \int_{0}^{\infty} \int_{0}^{\infty} y \mathrm{e}^{-\beta(x+y)} \mathrm{d} H(x, y)}{\beta \mu_{X} \mu_{Y}}
$$

and $V_{n}$ for $n \geq 1$ is defined as in (17). Then using Corollary 1 and formulae (30) and (31) of [9], we can prove that

$$
\mathrm{E}\left[D(t)^{2}\right]=2 \int_{0}^{t} \mathrm{E}[D(u)] \mathrm{d} u-\mu_{X} \mu_{Y}\left(\mathrm{E}[\tilde{N}(t)]+\mathrm{E}\left[\tilde{N}(t)^{2}\right]\right) .
$$

If $X_{1}+Y_{1}$ is a nonlattice random variable, then, using formulae (33) and (39) of [9] and (19) above, we obtain that, as $t \rightarrow \infty$,

$$
\begin{aligned}
\mathrm{E}\left[D(t)^{2}\right]= & \frac{\mu_{Y}^{2} t^{2}}{\left(\mu_{X}+\mu_{Y}\right)^{2}} \\
& -\left[\frac{\mu_{X} \mu_{Y}^{3}+\left(\mu_{X}^{2}-2 \sigma_{X}^{2}\right) \mu_{Y}^{2}+\left(\sigma_{Y}^{2}+4 \sigma_{X Y}\right) \mu_{X} \mu_{Y}-\mu_{X}^{2} \sigma_{Y}^{2}}{\left(\mu_{X}+\mu_{Y}\right)^{3}}\right] t+o(t),
\end{aligned}
$$

and hence, by taking (16) into consideration, the last part of the theorem follows.

Remark 3. The first result (15) of Theorem 2 can also be proved using a Tauberian theorem. From (3), if $\mu_{X}$ and $\mu_{Y}$ are finite, we obtain that

$$
\int_{0}^{\infty} \mathrm{e}^{-\beta t} \mathrm{dE}[D(t)] \sim \frac{\mu_{Y}}{\beta\left(\mu_{X}+\mu_{Y}\right)} \quad \text { as } \beta \rightarrow 0 .
$$

Obviously $\mathrm{E}[D(t)]$ is nondecreasing. So we can use a Tauberian theorem (see Theorem 4.3 of [10]) to conclude (15).

Asymptotic distribution of the total downtime is given in the following theorem, which is a generalization of the results in [9] and [6].

Theorem 3. If $\sigma_{X}^{2}$ and $\sigma_{Y}^{2}$ are finite, then

$$
\frac{D(t)-\mu_{Y} t /\left(\mu_{X}+\mu_{Y}\right)}{\sqrt{\left(\left(\mu_{X}^{2} \sigma_{Y}^{2}+\mu_{Y}^{2} \sigma_{X}^{2}-2 \mu_{X} \mu_{Y} \sigma_{X Y}\right) /\left(\mu_{X}+\mu_{Y}\right)^{3}\right) t}} \stackrel{\mathrm{D}}{\rightarrow} N(0,1) \text { as } t \rightarrow \infty .
$$

Proof. First note that

$$
\sum_{i=1}^{N(t)} Y_{i} \leq D(t) \leq \sum_{i=1}^{N(t)+1} Y_{i}
$$


where $N(t)=\sup \left\{n \geq 0: \sum_{j=1}^{n}\left(X_{j}+Y_{j}\right) \leq t\right\}$. Using the central limit theorem for random sums (see [2]), we obtain that

$$
\left[\frac{\mu_{X}^{2} \sigma_{Y}^{2}+\mu_{Y}^{2} \sigma_{X}^{2}-2 \mu_{X} \mu_{Y} \sigma_{X Y}}{\left(\mu_{X}+\mu_{Y}\right)^{3}} t\right]^{-1 / 2}\left(\sum_{i=1}^{N(t)} Y_{i}-\frac{\mu_{Y} t}{\mu_{X}+\mu_{Y}}\right) \stackrel{\mathrm{D}}{\rightarrow} N(0,1) .
$$

The proof is complete if we can show that

$$
\frac{Y_{N(t)+1}}{\sqrt{t}} \stackrel{\mathrm{P}}{\rightarrow} 0 \quad \text { as } t \rightarrow \infty .
$$

But by the fact that $N_{\mathrm{p}}(t) / t \stackrel{\mathrm{P}}{\rightarrow} 1 /\left(\mu_{X}+\mu_{Y}\right)(>0)$ and the assumption that $\sigma_{Y}^{2}<\infty$, we obtain that $Y_{N(t)} / \sqrt{N(t)} \stackrel{\mathrm{P}}{\rightarrow} 0$ (see e.g. Lemma 3 of [6] for an argument), and hence the required statement follows.

\section{An example}

In this section we give an example to see the effect of dependence of the failure and repair times on the distribution of the total downtime. Suppose that the failure time $\left(X_{i}\right)$ and the repair time $\left(Y_{i}\right)$ have a joint bivariate exponential distribution given by

$$
\mathrm{P}\left(X_{1}>x, Y_{1}>y\right)=\mathrm{e}^{-(\lambda x+\mu y+v \max (x, y))}, \quad x, y \geq 0, \lambda, \mu, v>0 .
$$

In this case, both $X_{1}$ and $Y_{1}$ are exponentially distributed with parameters $\lambda+v$ and $\mu+v$ respectively, and hence

$$
\begin{aligned}
\mu_{X} & =\frac{1}{\lambda+v}, & \mu_{Y} & =\frac{1}{\mu+v}, \\
\sigma_{X}^{2} & =\frac{1}{(\lambda+v)^{2}}, & \sigma_{Y}^{2} & =\frac{1}{(\mu+v)^{2}} .
\end{aligned}
$$

The covariance and correlation coefficient between $X_{1}$ and $Y_{1}$ are given by

$$
\sigma_{X Y}=\frac{v}{(\lambda+\mu+v)(\lambda+v)(\mu+v)} \quad \text { and } \quad \rho_{X Y}=\frac{v}{\lambda+\mu+v}
$$

respectively.

The effect of dependence between the failure and the repair times can be seen in Figure 1. In this figure we compare the graphs of the normal approximations of $D(10)$ where $\left(X_{i}\right)$ and $\left(Y_{j}\right)$ are independent and both $X_{1}$ and $Y_{1}$ are exponentially distributed with parameters $\lambda+v$ and $\mu+v$ respectively with the normal approximations of $D(10)$ for various correlation coefficients $\rho_{X Y}$.

In this example we can also calculate explicitly the mean of the total downtime $D(t)$. Using (3), we obtain that

$$
\int_{0}^{\infty} \mathrm{E}[D(t)] \mathrm{e}^{-\beta t} \mathrm{~d} t=\frac{(2 \lambda+v) \beta+(\lambda+v)(\lambda+\mu+v)}{\beta^{2}\left[2 \beta^{2}+(3 \lambda+3 \mu+4 v) \beta+(\lambda+\mu)(\lambda+\mu+3 v)+2 v^{2}\right]} .
$$

This transform can be inverted analytically. As an example, for $\lambda=1, \mu=2$ and $v=3$, we obtain that

$$
\mathrm{E}[D(t)]=\frac{4}{9} t-\frac{13}{162}+\frac{2}{81} \mathrm{e}^{-9 t / 2}+\frac{1}{18} \mathrm{e}^{-6 t} .
$$


(a)

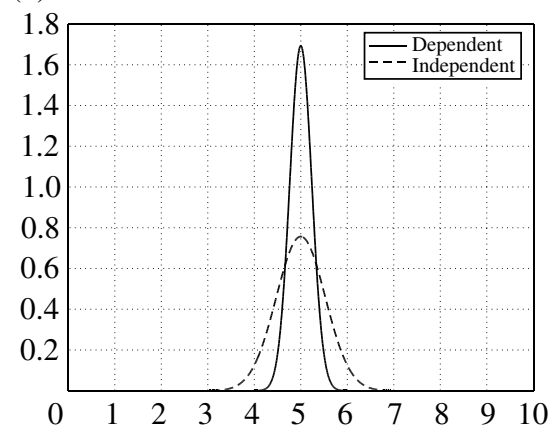

(b)

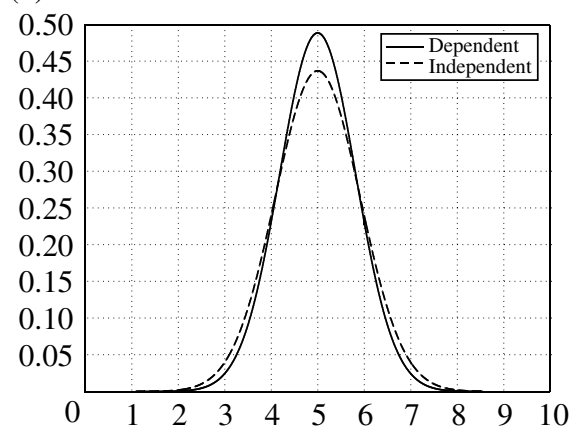

FIGURE 1: The graphs of the normal approximations of $D(10)$ with (a) $\rho_{X Y}=0.8(\lambda=\mu=1, v=8)$ and (b) $\rho_{X Y}=0.2(\lambda=\mu=2, v=1)$. Solid line for dependent cases and dashed line for the independent cases.

\section{References}

[1] Barlow, R. E. and Proschan, F. (1975). Statistical Theory of Reliability and Life Testing. Holt, Rinehart and Winston, New York.

[2] Embrechts, P., Klüpelberg, C. And Mikosch, T. (1999). Modelling Extremal Events for Insurance and Finance. Springer, Berlin.

[3] Funaki, K. AND Yoshimoto, K. (1994). Distribution of total uptime during a given time interval. IEEE Trans. Reliab. 43, 489-492.

[4] Grandell, J. (1943). Doubly Stochastic Poisson Processes. Springer, Berlin.

[5] Muth, E. J. (1968). A method for predicting system downtime. IEEE Trans. Reliab. 17, 97-102.

[6] RÉNYI, A. (1957). On the asymptotic distribution of the sum of a random number of independent random variables. Acta Math. Acad. Sci. Hungar. 8, 193-199.

[7] Resnick, S. I. (1987). Extreme Values, Regular Variation, and Point Processes. Springer, New York.

[8] Srinivasan, S. K., Subramanian, R. and Ramesh, K. S. (1971). Mixing of two renewal processes and its applications to reliability theory. IEEE Trans. Reliab. 20, 51-55.

[9] TAKács, L. (1957). On certain sojourn time problems in the theory of stochastic processes. Acta Math. Acad. Sci. Hungar. 8, 169-191.

[10] WidDER, D. V. (1946). The Laplace Transform. Princeton University Press. 